\title{
APCOL: A programming system for computer-controlled psychology laboratories*
}

\author{
ALLEN L. PINKUS $†$ and LEE W. GREGG \\ Carnegie-Mellon University, Pittsburgh, Pennsylvania 15213
}

The development of on-line computer systems can be facilitated by higher-level programming languages that are more powerful and easier to learn than machine-level Assembly languages. In general, it is not possible to speak of $a$ programming language without also describing the operating system and functional problem domain of the small computer system for which it is intended. This is because of the great variety in the architecture and configuration of small computers. However, it is possible to state more general requirements and design criteria for on-line computer languages in psychology. APCOL is an example of an integrated programming system. One of the important distinguishing features of the system is that it is implemented both on the small-scale laboratory computer and on a large general-purpose computer linked to the smaller machine via a high-speed communications network. This arrangement permits firsthand access to interactive program construction with immediate test of program function by using the on-line implementation as well as access to the text-editing and mass storage facilities of the large-scale machine.

It has now been a decade $\mathrm{c}^{r}$ so since process-control minicomputers became widely available to behavioral science laboratories. During this period there has been a great deal of speculating, and actual reporting, on the tremendous benefits to science and education possible within the computer-controlled psychological laboratory (CCPL). The bulk of these papers have appeared in previous issues of this journal. Computer manufacturers have certainly demonstrated that significant price reductions can be offered even while advances are made in hardware capabilities and system sophistication. Systems availability is consequently very high. In contrast, we have not seen a concerted effort by

\footnotetext{
*Development of the APCOL language was supported by the Public Health Service, National Institutes of Health, Grant GM 16716. The computer-controlled psychology laboratory at Carnegie-Mellon University has received continuing support from the Public Health Service, National Institute of Mental Health, Grant MH 07722.

tAllen L. Pinkus is now associated with Applied Science Associates, Inc., Box 158, Valencia, Pennsylvania 16059.
}

manufacturers, or user groups, to develop and standardize a programming language designed for the wide variety of experimental paradigms and tasks which characterize modern psychology, certainly not to the extent that language development was supported for early general-purpose computers. CCPL language evolution has occurred largely on a user-independent basis, and there has been little reported effort to relate characteristics of the psychological task domain to the design of a CCPL language. As a result, the existing languages and programming systems are rather varied in character and limited to particular user needs. It is not unreasonable to suggest that most CCPLs have not lived up to their initial expectations of providing research and educational contributions, and we believe that the language barrier is the primary cause of this problem. In 1970 the problem clearly existed at the CCPL at Carnegie-Mellon University. Even though this installation possessed a rather advanced hardware configuration and system supervisor and support programming, the Carnegie-Mellon University CCPL was not experiencing high user acceptability and system utilization was low. A project was therefore initiated to develop a high-level language and programming system to satisfy general user requirements in a "human factored" way and also to capitalize on the available computing resources at the university. This paper summarizes the outcome of that project.

\section{THE CARNEGIE-MELLON UNIVERSITY CCPL}

The Carnegie-Mellon laboratory and supporting facilities are depicted in Fig. 1. The laboratory is designed around a Honeywell DDP-116 16-bit computer with $16 \mathrm{~K}$ of core memory organized into 32512 -word sectors. The machine has a priority interrupt system, a real-time clock running in milliseconds, and assorted electronic enhancements for the support of a multidevice multiuser environment. System and user programs are stored on a direct-access 6-megabit fixed head disk. Communication with experimental Ss is accomplished through seven independent "room 


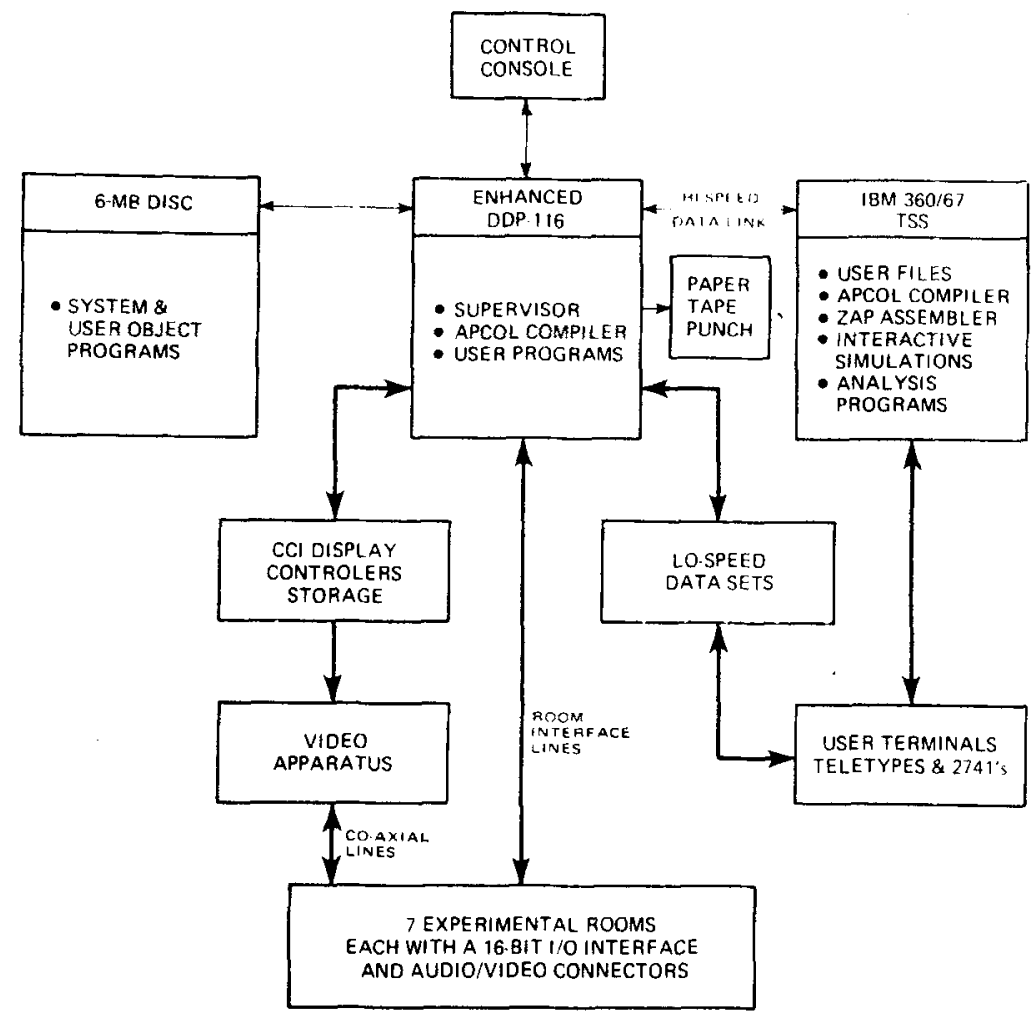

Fig. 1. The Carnegie-Mellon CCPL environment.

interfaces," which essentially are extensions of the 16-bit $\mathrm{I} / \mathrm{O}$ bus, through dataset-coupled Teletypes, and through a visual display system consisting of two $\mathrm{CCl}$ character gencrators (each adding $1 \mathrm{~K}$ half-words of memory) tied to composite video generators, video switchers, monitors, and a VTR. User communication with the DDP is through a Delta Data Systems TelTerm video console. Data sinks are provided by the Teletypes, a high-speed paper tape punch, and the system disk. A high-speed data link ties the DDP to the IBM 360/67 maintained by the Carnegie-Mellon University Computation Center. Operations on this machine are supervised by an enhanced variant of IBM's time-sharing system TSS/360. User terminals in the laboratory area provide access to TSS. CCPL activities are controlled by FIASCO ${ }^{1}$ a time-sharing monitor specifically oriented toward CCPL applications. FIASCO handles all the intricacies of $\mathrm{I} / \mathrm{O}$, interrupt servicing, and time slicing and allows users to load, unload, edit, and execute programs in the time-shared environment. User programs acquire their real-time capabilities via call to reentrant macro routines. FIASCO requires $8 \mathrm{~K}$ of memory for the core-resident control routines and macros and the areas into which transient macros and console routines are loaded from the disk when required.

\section{Programming Developments}

Prior to 1971 , the primary programming support for the CCPL was an Assembly code and macro language (ZAP) and an assembler resident on the Carnegie-Mellon University Univac 1108. Control programs were punched on cards, assembled, and transferred to the CCPL via paper tape. The disadvantages entailed by this procedure were numerous. First, the entire operation was very time consuming: it typically required at least a week to bring a program up to initial check-out status. The time and effort interfered significantly with both educational objectives and research plans. Secondly, Assembly code is not a good vehicle for expressing complex experimental procedures. It generally takes a long time to learn, to program in, and to debug.

The project designed to alleviate these difficulties consisted of two distinct phases: (1) the development of a process-control language for CCPLs and (2) the implementation of the language within a programming system to maximize its availability to multiple users. Some design criteria for this programming system were (1) the provision for rapid turnaround time so that programmers could evolve and validate a useful program quickly, (2) a compatibility with existing systems that users already knew or would learn concurrently, (3) the full utilization of available power of the university computers, and (4) a provision for hands-on programming and program execution to insure the system's motivational and educational value. As will be discussed later, the design of the language evolved somewhat differently: it was to be built up out of studies of the CCPL environment, its targeted users, and the structure of psychological experimentation.

\section{Total System Overview}

The CCPL language developed in Phase 1, APCOL, ${ }^{2}$ 
exists as two separate implementations. The first is entirely written in ZAP and runs under executive control on the DDP-116. Users write programs on-line und can have them executed immediately. Compilation is on a statement-by-statement basis, and no source code 's retained. Vonetheless an editing teature is muiahle correct tatements during somplation or hes

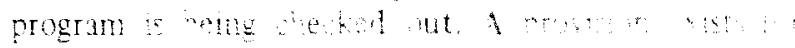

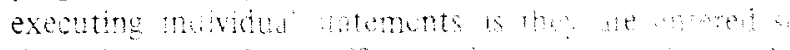

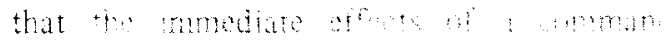

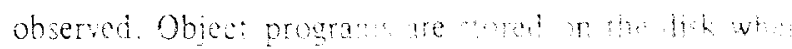

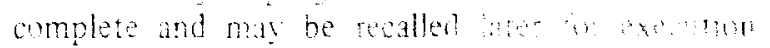

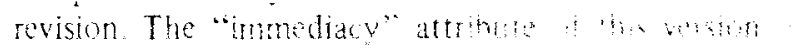

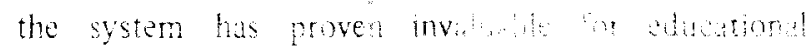

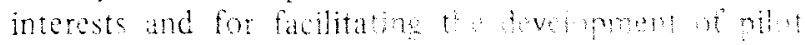

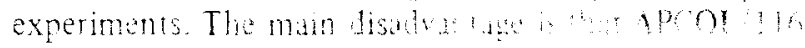

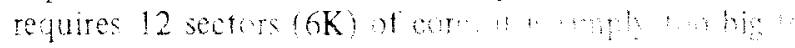
be used routinely when other as aro nom

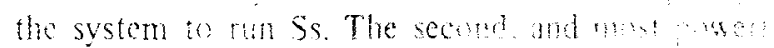
version of the APCOL stom wasta

FORTRAN-coded comple: and ZAP assentlor m IBM 360/67. Users write or revise programs under a $\mathrm{S}$ editor and call the APCOI minessor in is mith identical with : 2 way whih other TSS langu

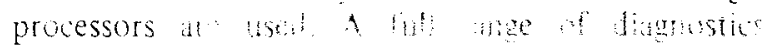

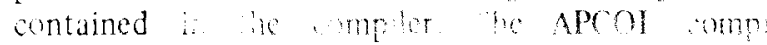
produce Al' cude which an an inticaly passed to

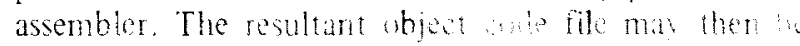
trans:aited to the DDP wia the high eed dita link :It of tha intricacies of this process are embetded in $\mathrm{T}_{\mathrm{s}}$ provedure definition (PROCDFFil and is appars you traisparent to the user. The major disadvantage of the system is that ideal turnaromnd time s increased in m hour or so. However. this is more than compensated sin by the powertul task and Aata mangement acilities of TSS and by the fact that the COP! is released for ts primary function A brief sketch of some internals of these APCOL systems will be presented later on.

\section{Language Overview}

The APCOL language itselt can best be described as procedure oriented. The procedures or commands were designed and selected with several criteria in mind. First of all. the language was to be easy to learn and easy to use - "natural" for its intended application. Secondly. it was to be usable as a vehicle for clearly conveying the essential ingredients of an experiment between people. For training purposes, program "readability" was to be high. Thirdly. it was to be compatible. where possible, with the features of other languages commonly in use (FORTRAN and PL/1). Finally. it was to make clear to the user the attributes of real-time process control. including interrupt handling. time period waits and measuring. and device control. A guiding philosophy we maintained was that the language should be able to handle a wide rariety of experimental tasks and not be tied down to a particular paradigm. such as operant conditioning. The design criteria were met. we believe. by building a command repertoire which directly associates ratements with (1) the types of component tasks that anologists perform in the laboratory and ulk ablt heir research and the generic types of aprarat: an ase and $(2)$ the types of operations that ill are are ortorming and that

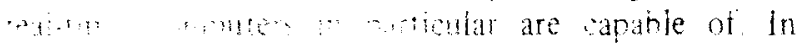

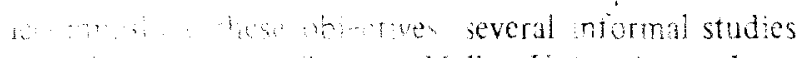
a w allon Unversity students

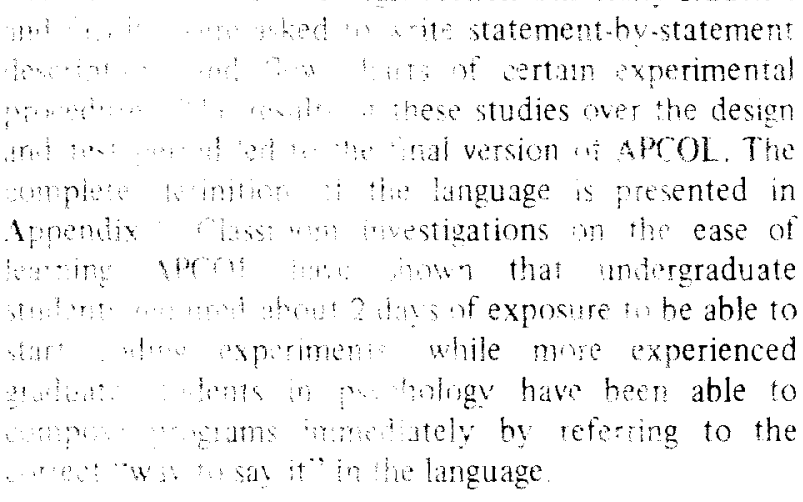
THF APCOL LANGUAGE

The 5 ammand in APCOL can be broken down in it: a lain categories. First, there

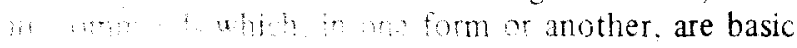
mi namming lanages. Second, there are

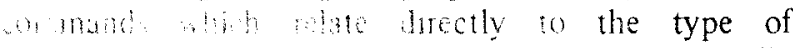

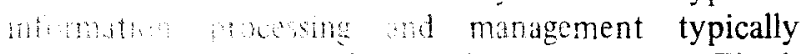

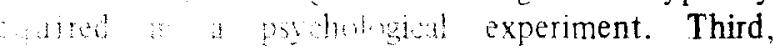

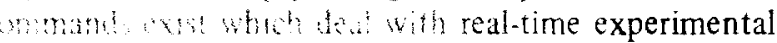
ontrol. Thime molude statenents allowing control over S.cumpute weraction afd statements for experimental abparatus tandling Thes subsets will be briefly discussed the tollow sections. ${ }^{3}$ See the syntax for each in Aptratix!

\section{Basic Programming Commands}

Console 10: "Console" permits experiment status information to be displaved on the DDP console ("console subiect now in rest period""). "Read" allows aphanumicric sharacters :r numeric values, such as experimental parameters. to be entered at run time ("read 5 numbers into list parameters"). During compilation. the read instruction allocates the required number of words for storing the list.

Basic arithmetic and information transfer: "Store" performs (integer) arithmetic computations and single value data transters ("store response "60 in response"). "Select" and "change" access or modify values within lists ("select word item word from list words," "change item 1 on list historic to newral").

\section{Transfer of Control}

"If" permits branching contingent upon the truth of a relation between two terms. Besides numerical comparisons, it is possible to test for the presence of information on lists ("if response is on stimuli goto 
correct"). "Goto" simply forces the transter of control to a specified statement.

\section{Process Structures}

Iterative loops are defined by "begin loop" and "end loop" statements. Within these loops, the positively increasing value of the index variable is accessible by other commands for indirect addressing or other purposes ("begin loop display do stim\# from 1 to n"). In addition to iterative structures, it is possible to define blocks of statements which are executed only under certain specified conditions. The start of these blocks is delimited by the "begin procedure" statement, the end of the block by "end procedure" ("begin procedure training do until \#correct gt criterion"). Finally, blocks of statements called "tasks" can ue defined which are executable from several places within a program. They are like subroutines whose symbol table is shared by the main program.

All information within an APCOL program is contained within structures called lists. "List" seemed to be an appropriate name for these structures because psychologists typically deal with them (e.g., lists of S.R pairs) and also because there are many experiments in which information is transient (i.e., it gets created, used, and then destroyed). There are several different commands for manipulating these structures.

\section{List Definition and Organization}

"Reserve" is a nonexecutabie command which allocates a specified amount of space for a list used in an experiment ("reserve 450 locations for list population"). The "read" command performs the same function. Since psychologists commonly work with stimulus items that are larger than one character, the "group" command is available to define the size of the basic unit on a list. In the language, these basic units are called "items." In a learning experiment, for instance, an item may be three characters long, such as a CVC. If these were read or generated into list population, the statement "group list population by 3" would allow all other commands to access the CVCs on the list and not individual characters.

\section{Randomization Commands}

Because randomization is an integral part of so much psychological research, three commands have been included in APCOL to produce and manipulate lists in random ways. "Generate" simply provides a single random integer ("generate a random number stim 1 to n.stim"). "Permute" randomly reorganizes the items in a list. From the preceding example, "permute list population" would reorder the CVCs on the list and not the characters. Finally, the "produce" command generates a random sample of items with or without replacement from some available population. A list of CVCs may be created for a particular trial by stating, for example. "produce 10 random.n. items sampl from list population."

\section{Dinamic List Manipulation}

Often in experiments the size of lists may be undefined when the control program is written. The size may be contingent on some experimental parameter or on the performance of the S (i.e., the number of errors he makes during learning). "Add." "delete," and "empty" control the size and contents of lists. Examples are "add error to list errors," "delete response from list sample," and "empty list temporary." In the case where the number of items on a list is unknown. "count" fetches that value ("count list errors in nerror"). The "shift list" command moves items up or down within a list. A case in which this procedure is appiicable is when historic lists are kept ("shift list historic down to oldest"). Finally, simple statistics (means and sums of squares) may be computed from a list of data and used in the control of an experiment ("compute xbar mean of list errors").

\section{Real-Time Control Statements}

While the preceding types of commands may be found in any computer language, there are operations performed in the CCPL that are unique to real-time environments. The syntax of these statements attempts to reflect what psychologists typically do when instructing a machine or a research assistant in interacting with an experimental S.

\section{Basic Interaction and Timing}

"Set" permits a program to exercise control over the output state of the experimental room interface. Bits may be designated to be turned on or off to activate small-load relays or lights. The binary pattern contained in the command's argument appears at the interface until changed or until the optional time period expires. For example, "set bit $\$ 5$ for duration ms" may control the shutter of a slide projector (the shutter circuitry being connected to bit 5 , a mapping that the user must decide in advance). The "wait" command has several variations. The execution of a program can be suspended for a certain number of milliseconds ("wait $1500 \mathrm{~ms}$ ") or until an interrupt is generated by a piece of apparatus connected to an experimental room interface ("wait for any response answer"). The allowable interrupting bits are specified in the statement syntax. The two cases can be combined as in "wait pause ms or for bit ' 377 response rl." The "wait" command can also be used to handle data from a real-time eye-movement digitizer which connects to a room interface. Whenever an eye movement is detected by this device, an interrupt is generated and the position of the eye spot is available to the program. This position is coded in a format identical with that used for displaying information on the $\mathrm{CCI}$ display system (described below). Complex interactive eye-movement experiments can therefore be performed 
quite easily. An instance of this feature is "wait criterion $\mathrm{ms}$ or for coordinates $\mathrm{x} y$." Communications with a $\mathrm{S}$ at a dataset-coupled Teletype can be accomplished with the "ds" command. Information can be sent to the Teletype ("ds 1 out stimlist") and can be received as the S types ("ds 1 in respword"). Finally, "timer start" and "timer end" permit periods of time, with any intervening process, to be measured with millisecond accuracy (e.g., "timer end latency"). Besides measuring $\mathrm{S}$ response latencies, this function may be used to time complex stimulus generation procedures and subsequently adjust wait periods so that $S s$ are not cued with respect to the nature of the next stimulus presentation. The degradation in "real-time" control becomes obvious to users in this case.

\section{Display. Control}

"Display" transfers the contents of a list into the storage of the CCI controller where it may be presented to a $\mathrm{S}$ on a video monitor. Any complex display configuration consisting of integral characters can be handled by this command. Provisions exist for displaying numbers and specifically input sentences for complex language experiments. Examples are "display list.c. words at 10 row space by 2" and "display sentence nsent at column 5." "Blank" allows total or selective erasure of the CCI storage. "Refresh" controls the video generator section of the $\mathrm{CCI}$ controller. When "on," the contents of the $\mathrm{CCl}$ memory are presented on a monitor. Since this process is extremely fast when compared with information transfer between the DDP and CCI memories, complex tachistoscopic-like experiments can be run ("refresh on for $50 \mathrm{~ms}$ ").

\section{Data Recording}

Experimental "data sheets" are formatted and maintained through the use of the "record" statements. Data are transmitted to a Teletype or punch or saved on the system disk for transfer through the DDP-TSS network for subsequent analysis. Examples of the use of this command are "record 'trial stim resp latc" " and "record list.n. data."

\section{Network Communications}

Perhaps the most interesting aspect of the CCPL is the degree to which innovative experiments can be performed. It is possible, in the Carnegie-Mellon laboratory, to run experiment control programs on the DDP-116 and data analysis or simulation programs on the $360 / 67$ in parallel and have them interact. Using this facility. control programs can be modified in real time in many ways via changes in parameter settings or stimulus materials, dependent on the results of analyses done on another computer. It is even possible to load new control programs as they may be required. Conversely, a simulation running on TSS can be provided with data about an S's performance in a logically equivalent task so as to shape the model's characteristics to more

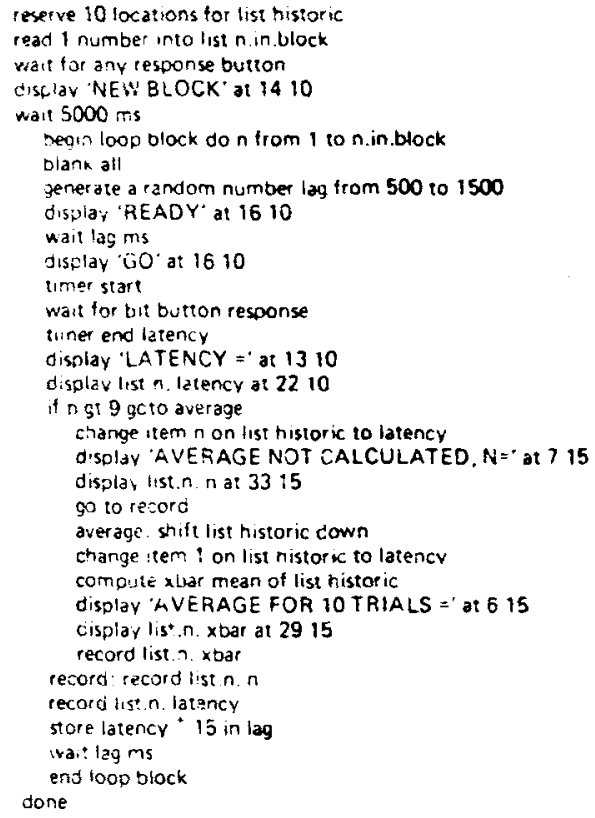

Fig. 2. An example of a simple APCOL program.

adequately fit the S's. These procedures are accomplished primarily through the "send" and "receive" statements, whose execution sequence must be made to properly interleaf with the execution of the TSS program. Several concept-learning experiments have already been performed with these features.

\section{Assembly Code Escape}

In the 360 version of APCOL, users are allowed to write Assembly code (ZAP) programs for special purposes such as high-speed calculations or display functions. For these applications, the "zap" command provides the control linkage and data transfer structures between the APCOL program and the ZAP code. Two registers are available for $1 / 0$ communications. An example is "zap modulo $n$ mod result."

\section{APCOL EXAMPLES}

The following program (Fig. 2) measures a S's response to a visual display. This example is provided not only to show an intact example of the language, but also to allow its comparison with PSYCHOL, another language also developed at Carnegie-Mellon University (McLean. 1969). While the function of this program should be reasonably clear, a more detailed description appears in the McLean paper (p.323). Except for educational purposes, programs of this size and complexity are not created by the APCOL/116 system.

A further example of APCOL programming is shown in Fig. 3. This program is for Sternberg's (1966) experiment in which memory set size was varied and Ss were cued for the correct response key just prior to the presentation of the probe or test item.

At execution time, the program generates a read request for 10 numbers. the stimuli to be presented on a 


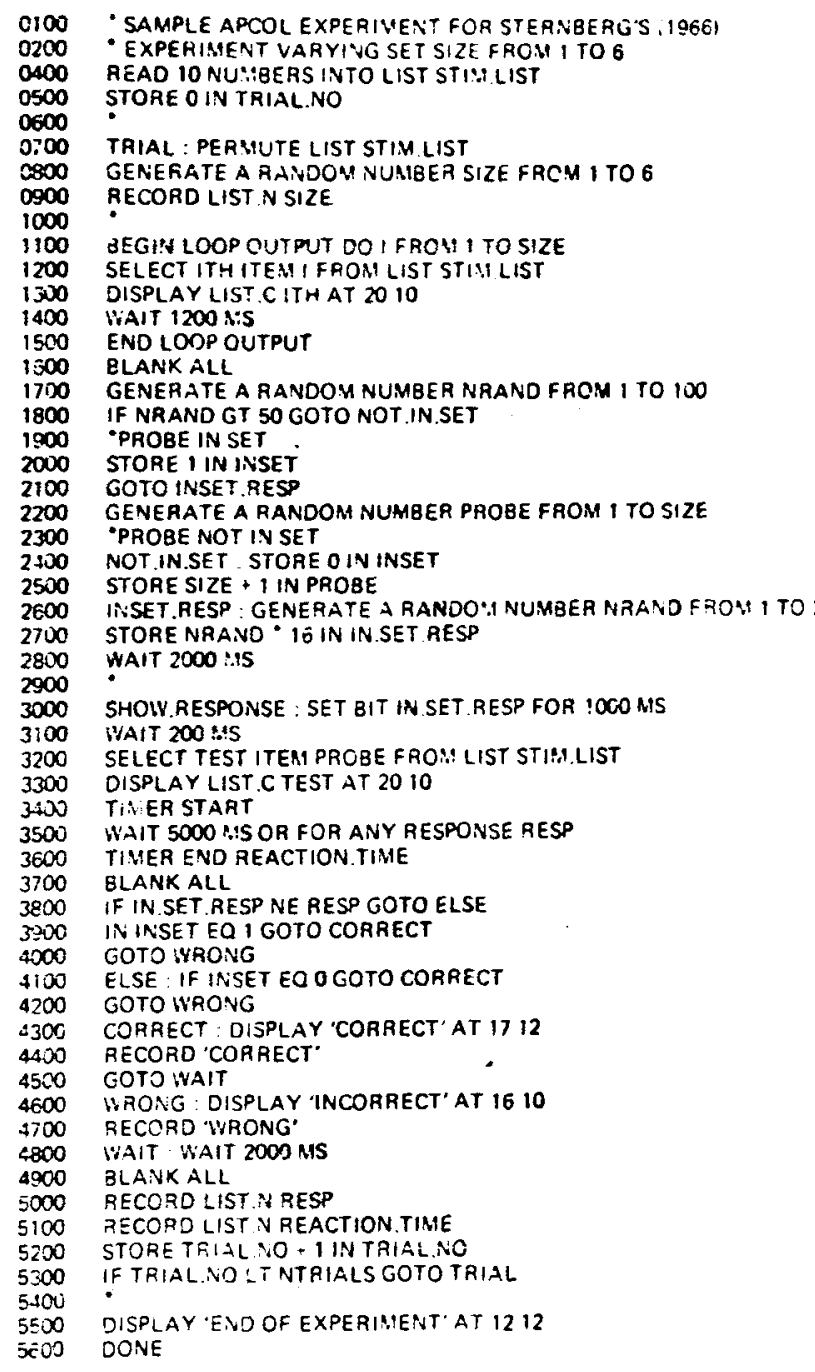

Fig. 3. APCOL program for Stemberg's (1966) experiment.

display screen. On each trial, this list is permuted to provide a random ordering of the numbers. Then a randomly selected value of the memory set size is generated and recorded. Notice that the ability to use mnemonic labels greatly facilitates the readability and intelligibility of APCOL programs.

The loop that begins at Line 1100 presents the memory set to the S. Each of the numbers in the set is shown at the same location on the screen for a period of $1,200 \mathrm{msec}$. The construction using the select instruction picks items one at a time from the stimulus list for display. Since the items are alphanumeric, the display instruction specifies that the list of items is represented in the character code, "Display list.c ITH at 20 10." If conversion of integer numeric values were required, this instruction would have been, "Display list.n ITH at 2010 ." Lines 1700 to 2800 generate the probe or test item, randomly selecting probes that are either in the memory set or not. with equal probability. Following the $2,000-\mathrm{msec}$ wait, the delay between presentation of the memory set and the warning signal, the probe item is presented. The reaction time of the $S$ is obtained in Lines 3400 to 3600 . Finally, the appropriate feedback is given.

\section{INTERNALS}

It should be apparent from the example where the major restriction is in the language and why programs often become lengthy. The format of terms or arguments in APCOL expressions are constrained and commands cannot be nested within others. For example, it is not possible to say "wait latency * $15 \mathrm{~ms}$." These abilities were traded off against gains in compiler efficiency and object code transparency (i.e., the object code is understandable). In both versions of APCOL, the processing of source statements results in three separate sections of code in the object programs. First, all variables, constants, and strings are given an argument code and inserted in the data region. The code of a list is followed by a word containing the item size (established by the "group" command or defaulted as " 1 ") and the number of single words in the list. The purpose behind coding each data entry and not using (relocatable) addresses is that lists may move about in the data region when the "add." "delete," or "empty" statements are executed. A search process locates data by accessing the argument codes and skipping over the other entries. The second section in an object program is called a program segment. This segment consists of a jump instruction followed by a fixed number of argument codes, one for each argument in a command. "Produce," for example, has four arguments: the code for the list containing the number of items to produce, a 0 for replacement or a 1 for nonreplacement sampling, and two more argument codes for the sample list and the population list. The third section consists of prewritten blocks of ZAP code, similar to macro routines, that perform the process specified in the APCOL source statement.

Control sequencing is accomplished in different ways for the two versions of the system. In APCOL/116, a control module fetches the address of a program segment from a table and passes control to that address. The address contains an indirect jump through a transfer vector (a table of address constants) whose values are the addresses of the initial instruction in the appropriate execution module. Control returns from that module to the sequencer. Argument codes are retrieved by maintaining a record of the address of the word following the jump instruction. In APCOL/360 there is no need for external control sequencing because all addresses are assigned by the ZAP assembler. The jump instructions in the program modules are "JSTs" (jump and store) which directly address the appropriate execution module. The return address from a JST is incremented by the number of arguments in the program segment so that control returns to the next executable instruction. This may be another JST, a single instruction like "JMP" (corresponding to a "goto" statement), or a single word ZAP macro call, such as 
Fig. 4. Data and control structures of APCOL object programs.

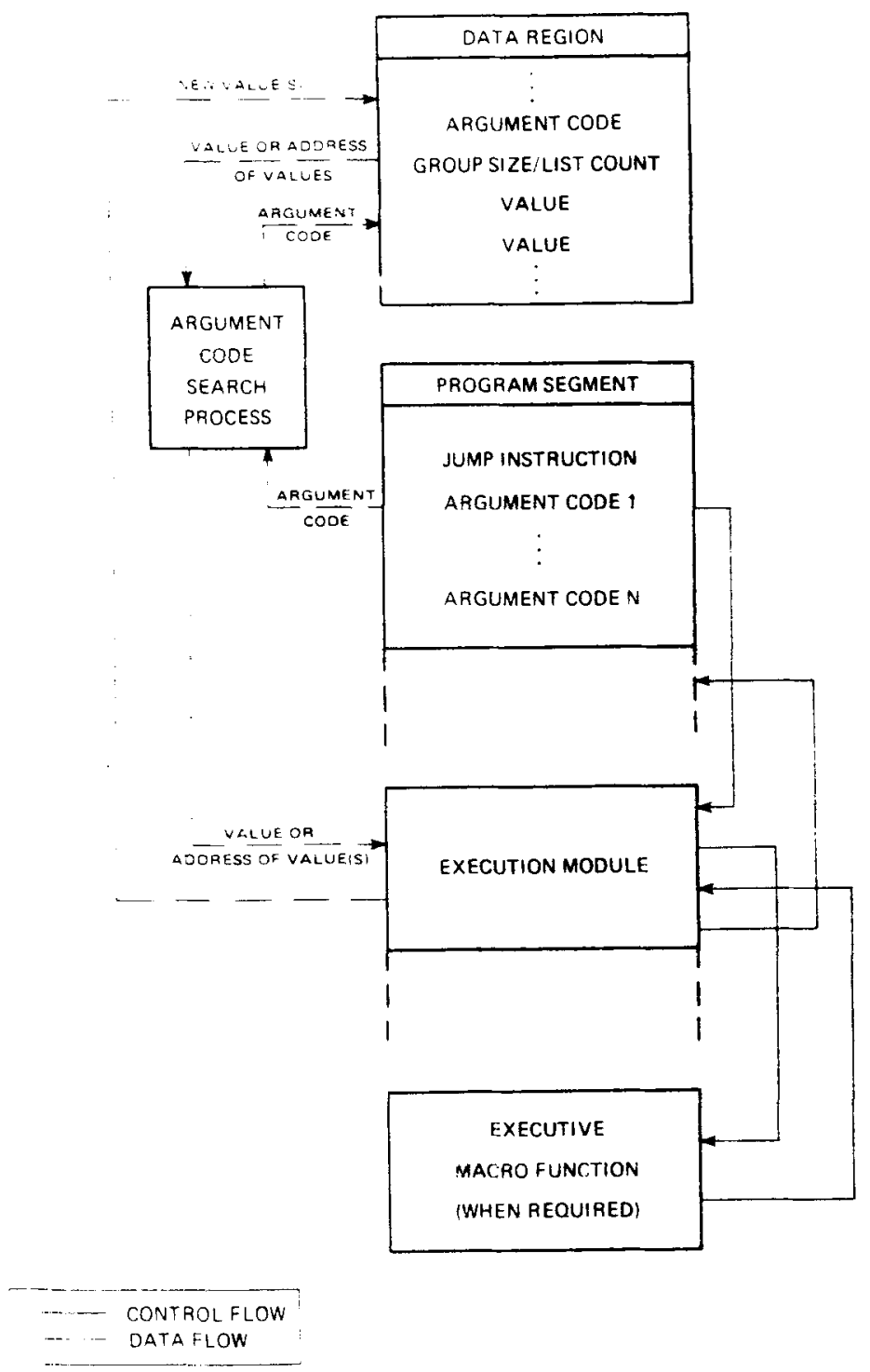

"BLK" (associated with the APCOL statement "blank all").

Because the APCOL/116 system is preassembled, it requires a large amount of core for compilation and execution (6 or 7 sectors). The APCOL/360 system, in contrast, produces much more tailored and efficient code. A very large APCOL/360 program (200 lines or more) might require 6 sectors of core. Plans have been made to reduce this requirement by writing the APCOL execution modules as reentrant macros in the core-resident operating system FIASCO. This would allow very large source programs to occupy only a small partition of user core and would consequently enhance the multiple-user aspect of the CCPL.

\section{SYSTEM EVALUATION}

As far as we have been able to tell. APCOL, as a programming language for CCPLs, stands up quite well with respect to meeting our initial design criteria. APCOL is reasonably easy to learn and use and has shown no sign of being unable to express the most complex experimental situation. (The only exception may be its inability to handle multiple, hierarchically organized interrupts, but this feature is unavailable to user programs running under FIASCO. When new commands are desired or new systems become available. however, it is relatively easy to include them in the modularly designed compilers.) The language flexibility has allowed programs to be written for a variety of research projects on learning. perception, child development. animal training, and complex cognitive performance. Several real-time simulations (e.g.. a radar operator's task) and game-playing programs have also been run. Users have thus been able to explore many experimental paradigms and have avoided becoming trapped in any specific one. as is possible with the commonly used finite state languages.

The programming systems have also functioned well. and we believe that an approach which utilizes and integrates the available computing power is a worthwhile 
one to follow. During the initial phases of acquiring a CCPL, a great deal of system development can be performed long before the process-control machine arrives. Perhaps the performance of the system can best be summed up by relating that, on several ideal occasions. a user thought of an experiment one evening. programmed it the next morning, and had analyzed data to think about that night. This rapid turnaround for scientific hypothesis testing can be achieved, we believe. when the right set of tools are available.

\section{REFERENCES}

McLean, R. S. PSYCHOL: A computer language for experimentation. Bebavior Research Methods \& Instrumentation, $1969,1,323-328$.

Pintus, A. L. APCOL: Programmers reference manual. Department of Psychology, Carnegie-Mellon University, A pril 1972.

Stermberg, S. High-speed scanning in human memory. Science, $1966,153,652-654$.

\section{NOTES}

1. FIASCO, an acronym for "For Implementation and Support of Computer Operations," was developed and inappropriately named by Henry $H$. Mashbum, the CCPL systems programmer.

2. The expansion of this acronym has been forgotten. Some people believe that it reflects the first author's ego involvement with the system.

3. An extensive description of the language is presented in the APCOL Programmers Reference Manual (Pink us, 1972). This document is available to interested parties from the Camegie-Mellon University CCPL.

\section{APPENDIX 1}

The Definition of APCOL

APCOL: The Language Structures

The BNF definitions for the classes of operands allowable in $\dot{A} \bar{Y} \mathrm{COL}$ statements.

\section{General Classes}

$<$ symbol $>::=<$ letter $>\mid<$ symbol $><$ symbol character $\rangle$

$<$ symbol character $>::=<$ letter $>\mid<$ digit $>\mid<$ special character $>$

$\langle$ string $\rangle::=<$ symbol character $>\mid<$ string $\rangle\langle$ symbol character $>\mid<$ string $><$ string $>$

$<$ term $>::=<$ defined symbol $>\mid<$ integer $>\mid<$ octal integer $>\mid<$ bit $\rangle$

$<$ integer $>::=<$ digit $>\mid<$ integer $><$ digit $>$

$<$ octal integer $>::=$ '<octal digit $>\mid<$ octal integer $><$ octal digit $>$

$<$ digit $>::=<$ octal digit $>1819$

$<$ octal digit $>::=0|1| 2|3| 4|5| 6 \mid 7$

<letter $>::=$ alb $|c| d|e| f|g| h|\mathbf{i}| j|k||\mathbf{m}| \mathbf{n}|\mathbf{o}| \mathbf{p}|\mathbf{q}| \mathbf{r}|\mathbf{s}| \mathbf{t}|\mathbf{u}| \mathbf{v}|\mathbf{w}| \mathbf{x}|\mathbf{y}| \mathbf{z} \mid$

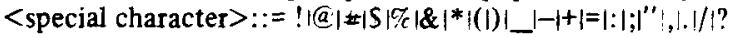

$<$ bit $>::=\$<$ integer $>$

$<$ list $>::=$ a data structure named by $<$ symbol $>$

$<$ d-symbol $>::=<$ defined symbol $>$

$\langle u-s y m b o l>::=<$ undefined symbol $>$

$<$ lable $>::=<$ symbol>

$<$ labled statement $>::=<$ lable $>:<A P C O L$ statement $>$

$<A P C O L$ statement $>::=<$ command name> [key words and operands]

$<$ command name>::= reserve|read $\mid$. . . Idone

\section{Statement-Specific Closses}

$<\mathrm{I} / \mathrm{O}$ format $\rangle::=$ characters $\mid$ numbers $\mathrm{c} \mid \mathrm{n}$

$<$ arithmetic expression $\rangle::=\langle$ term $\rangle \mid<$ term $\rangle$ <arithmetic operator $><$ term $>$ laddress of $<$ term $>$

$<$ arithmetic operator $>::=+|-| * !$

$<$ relational operator $>::=$ eqine $1 \mathrm{t}$ igt $t$ is on is not on

$<$ vertical direction>::= up!down

$<$ horizontal direction $>::=$ left $t$ right

<sampling procedure>::= replacement'non-replacement rin <execution condition $>::=$ when $/$ hile iuntil

$<$ statistic $>::=$ mean $\mid$ ss

$<$ refresh state $>::=$ onloff

$<$ allowable bits $>::=$ any $\mid$ bit $<$ term>

The definition of the syntax of APCOL statements.

reserve <integer > locations for list <symbol>

console '<string >'

read $<$ integer $><\mathrm{I} / \mathrm{O}$ format $>$ into list $<$ sumbol $>$

group list $<$ d-symbol $>$ by $<$ term $>$

store <arithmetic expression > in <symbol>

select $<$ sy'mbol $>$ item $<$ term $>$ from list $<$ d-symbol $>$

change item $<$ term $>$ on list $<d$-symbol $>$ to $<$ term $>$

add $<$ term $>$ to list $<$ d-symbol $>$

delete $<$ term $>$ from list $<$ d-symbol $>$

empty list $<\mathrm{d}$-symbol $>$

count list $<\mathrm{d}$-symbol $>$ in $<$ symbol $>$

shift list $\langle$ d-symbol $><$ vertical direction $>$ i to $\langle$ synbo $i\rangle]$

shift word <d-symbol $><$ horizontal direction $>$ [ to $<$ symbol $>$ ]

produce $<$ term $>$ random.<sampling procedure $>$. items

$<$ symbol> from list <d-symbol $>$

permute list <d-symbol>

generate a random number $<$ symbol $>$ from $<$ term $>$ to $<$ term $>$

begin loop <u-symbol $>$ do $<$ symbol $>$ from $<$ term $>$ to $<$ term $>$

[increment by $<$ term $>$ ]

end loop <d-symbol $>$

begin procedure <u-symbol> do <execution condition $>$ $<$ term $><$ relational operator $><$ term $>$

end procedure $<d$-symbol $>$

begin task <u-symbol>

end task <d-symbol>

do task $<$ d-symbol $>$

if $<$ term $><$ relational operator $><$ term $>$ goto <lable>lline number

goto $<$ lable $>$ line number

compute <symbol $><$ statistic $>$ of list $<$ d-symbol $>$

refresh <refresh state $>$ [fót $<$ temr $>$ mis?

display list. $<\mathrm{I} / \mathrm{O}$ format $>$. $<$ term $>$ at $<$ term $><$ term $>$ [space by $<$ term $>$

display '<string $>$ ' at $<$ term $><$ term $>$

display sentence $<$ term $>$ at $<$ term $><$ term $>$ [format $<$ term $>$ $[<$ term $>$ ]

blank all

blank $<$ term $><$ term $>$ [thru $<$ term $><$ term $>$ ]

wait for $<$ allowable bits $>$ response $[<$ symbol $>]$

wait <term> ms [or for <allowable bits> response [<symbol >]]

wait for coordinates <symbol $><$ symbol $>$

wait $<$ term $>$ ms or for coordinates $<$ symbol $><$ symbol $\rangle$

set bit $<$ term $>$ [for $<$ term $>\mathrm{ms}$ ]

timer start

timer end <symbol>

record list. $<\mathrm{I} / \mathrm{O}$ format $\rangle$. $\langle\mathrm{d}$-symbol $>$

record '<string $>$ '

record line

ds $\langle$ term $>$ in $\langle$ symbol $>$

ds $<$ term $>$ out $<$ d-symbol $>$

send list. $<\mathrm{I} / \mathrm{O}$ format $>$. $<\mathrm{d}$-symbol $>$ [record]

send '<string >' [ record]

send line [record]

receive list. <I/O format $\rangle$. <symbol>

break

zap <u-symbol> $[<$ term $>\quad[<t e r m>\quad \mid<s y m b o l>$ halt

$[<$ symbol $>]]]]$

done

Note: Brackets denote optional parts of statements. 